\title{
ABUSO, CONTROL Y VIOLENCIA EN LA PAREJA A TRAVÉS DE INTERNET Y LOS SMARTPHONES: CARACTERÍSTICAS, EVALUACIÓN Y PREVENCIÓN PARTNER ABUSE, CONTROL AND VIOLENCE THROUGH INTERNET AND SMARTPHONES: CHARACTERISTICS, EVALUATION AND PREVENTION
}

\author{
Manuel Gámez-Guadix', Erika Borrajo² y Esther Calvete ${ }^{2}$ \\ 'Universidad Autónoma de Madrid. ${ }^{2}$ Universidad de Deusto
}

La violencia en la pareja constituye un problema social de gran relevancia por su elevada prevalencia y por las consecuencias para sus víctimas. En los últimos años, las tecnologías de la información y de la comunicación, como Internet y los Smartphones, se han convertido en herramientas frecuentes para ejercer comportamientos de control y agresiones en las parejas jóvenes. En esta línea, la finalidad del presente trabajo es la de realizar una revisión actualizada sobre el abuso online en parejas jóvenes. Para ello, en primer lugar, se analiza en qué consiste este fenómeno y las principales manifestaciones del abuso online en la pareja a través de Internet y los Smartphones. A continuación, se lleva a cabo una revisión de los instrumentos desarrollados para su evaluación y sus propiedades psicométricas. Finalmente, se incluyen varias recomendaciones para el desarrollo de estrategias de prevención derivadas de la evidencia empírica disponible.

Palabras clave: Abuso online, Relaciones de pareja, Violencia en el noviazgo, Control, Cyberbullying.

Partner abuse is an important social problem due to its high prevalence and the consequences it entails for the victims. In recent years, information and communication technologies, such as the Internet and Smartphones, have become frequent tools for exercising controlling behavior and aggression in young couples. The purpose of this paper is to conduct a review of the research on online abuse in young couples. To this end, firstly, we analyze what this phenomenon consists of and the main manifestations of online abuse in the couple through the Internet and Smartphones. Then, a review is carried out of the instruments developed for the assessment of online abuse and their psychometric properties. Finally, several recommendations are outlined for the development of prevention strategies derived from the available empirical evidence.

Key words: Online abuse, Partner violence, Dating violence, Control, Cyberbullying.

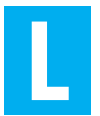

a violencia en la pareja constituye un problema social de primer orden por su elevada prevalencia (Vagi, Olsen, Basile y Vivolo-Kantor, 2015; Wincentak, Connolly y Card, 2017) y por las consecuencias para sus víctimas (Izaguirre y Calvete, 2015; Vu, Jouriles, McDonald y Rosenfield, 2016). Estas agresiones tienden a comenzar a edades tempranas durante las primeras relaciones de noviazgo (Bowen et al., 2014) y pueden constituir el precursor de conductas de abuso en etapas posteriores de la relación (Almendros, Gámez-Guadix, Carrobles, Rodriguez-Carballeira y Porrua, 2009). Los principales tipos de agresiones incluyen las físicas, las psicológicas y las sexuales (Almendros et al., 2009). Aunque tanto hombres como mujeres pueden ser víctimas de la violencia en la pareja, las mujeres son las que sufren las consecuencias más graves de estas agresiones (Archer, 2000).

En las últimas décadas, las tecnologías de la información y la comunicación (TICs), como Internet y los Smartphones, se han

Recibido: 15 julio 2016 - Aceptado: 5 julio 2018

Correspondencia: Manuel Gámez-Guadix. Universidad Autónoma de Madrid. Campus de Cantoblanco. 28049 Madrid. España. E-mail:mgamezguadix@gmail.com convertido en instrumentos habitualmente implicados en el desarrollo, mantenimiento y disolución de relaciones de pareja (Fox, Osborn y Warber, 2014). Además, las TICs son con frecuencia empleadas como medios para llevar a cabo comportamientos abusivos hacia la pareja, principalmente en forma de control psicológico y agresiones psicológicas y verbales (Borrajo, Gámez-Guadix y Calvete, 2015a; Zweig, Dank, Yahner y Lachman, 2013).

En este sentido, el abuso online en la pareja se ha definido como un conjunto de comportamientos repetidos que tienen como objetivo controlar, menoscabar o causar un daño al otro miembro de la pareja (Borrajo, Gámez-Guadix, Pereda y Calvete, 2015c; Reed, Tolman y Ward, 2017). Las conductas de abuso online a la pareja incluyen el control a través de las redes sociales, el robo o el uso indebido de contraseñas, la difusión de secretos o informaciones comprometidas, las amenazas y los insultos públicos o privados a través de las TICs. Estas conductas aparecen frecuentemente asociadas a comportamientos de agresiones psicológicas y físicas cara a cara, y podrían constituir un precursor de las mismas (Borrajo et al., 2015c). Además, según los estudios, el abuso online es habitual en las parejas. Los datos de prevalencia oscilan entre un 
$7 \%$ y un $80 \%$ (Hinduja y Patchin, 2011), dependiendo del tipo de abuso considerado.

La investigación ha mostrado que el abuso online en la pareja está asociado con una mayor depresión y ansiedad para las víctimas, mayor incertidumbre respecto a la relación, estilos de apego inseguro y ambivalente, comportamientos antisociales y mayores niveles de hostilidad, así como niveles de estrés percibido incluso mayores a los provocados por las agresiones tradicionales (p.ej., Hinduja y Patchin, 2011).

Por todo ello, se hace necesario avanzar en el conocimiento y prevención de este tipo de abuso en la pareja. En esta línea, el presente trabajo tiene los siguientes objetivos. En primer lugar, se analizan los aspectos relativos a las tipologías y la evaluación del abuso online en la pareja. Para ello, se describen las principales categorías de abuso online $y$, de manera relacionada, los instrumentos elaborados para evaluarlas. En segundo lugar, se revisan los aspectos referidos a la prevención del abuso online en las relaciones de pareja, incluyendo la necesidad de desarrollar enfoques holísticos y de trabajar sobre las actitudes que justifican la violencia. Finalmente, terminamos con una serie de recomendaciones y futuras líneas de investigación.

\section{TIPOLOGÍAS Y EVALUACIÓN DEL ABUSO ONLINE EN LA PA- REJA}

\section{Conceptualización y tipologías}

Si bien el estudio del abuso online en la pareja ha comenzado a generar mayor interés en los últimos años, aún son relativamente pocas las investigaciones realizadas sobre esta problemática (Brown y Hegarty, 2018; Hinduja y Patchin, 2011). Esta escasez de atención empírica ha derivado en la ausencia de una definición consensuada para delimitar el fenómeno, lo que ha generado una amplia variedad terminológica en lo referente a su conceptualización. En la Tabla 1 se incluyen las diferentes denominaciones propuestas por diversos autores, así como las definiciones o, en su caso, las conductas específicas que permiten caracterizar el fenómeno. Como puede observarse, algunos de los términos para referirse a este problema incluyen "abuso cibernético" en la pareja, "ciber agresiones", "violencia digital" en el noviazgo, "ciberacoso" en pareja, "agresiones electrónicas" o "victimización electróni$\mathrm{ca}^{\prime \prime}$, entre otros. A nuestro modo de ver, los términos abuso online en la pareja son los más inclusivos al dar cabida a un amplio rango de conductas, como el control psicológico (p.ej., dónde está la pareja en todo momento y con quién), el acoso (p.ej., llamadas repetidas e insidiosas) y las agresiones psicológicas y verbales, tales como insultos, amenazas y humillaciones (Borrajo y Gámez-Guadix, 2016; Zweig, Lachman, Yahner y Dank, 2014).

Las conductas de control o vigilancia a la pareja o expareja a través de medios electrónicos han sido los comportamientos que mayor interés han generado (Brown y Hegarty, 2018; Leisring y Giumetti, 2014). Zweig et al. (2013) encontraron que herramientas como el email, los teléfonos móviles, e incluso equipos como los GPS o las webcams, eran utilizados para llevar a cabo conductas de control en la pareja (p.ej., envío exce- sivo de e-mails, comprobación de llamadas y/o cuentas de correo electrónico o utilización de GPS, spyware, webcams y/o contraseñas personales para controlar a la pareja). Borrajo et al. (2015c), por su parte, examinaron las diversas formas de control y vigilancia a la pareja a través de redes sociales, como visitar frecuentemente el perfil de la pareja, leer los comentarios de sus amigos, revisar las fotos, las actualizaciones de estado y/o sus relaciones, o tratar de controlar al otro a través de su perfil en una red social. La prevalencia de estos comportamientos entre adultos jóvenes españoles fue del $75 \%$ para la perpetración y del $82 \%$ para la victimización.

Darvell, Walsh y White (2011) distinguieron los siguientes tipos de abuso: 1) Hostilidad electrónica, que incluye la publicación o envío de mensajes amenazantes, insultantes o dañinos a través de redes sociales, mensajes de texto o mail; 2) Intrusividad, referida al control de correo electrónico y redes sociales, cambio de contraseñas y creación de un perfil falso; 3) Humillaciones electrónicas, referidas principalmente a la publicación de fotos o información en redes sociales o webs para humillar - avergonzar a la víctima; y 4) Exclusión electrónica, referida a la eliminación, exclusión o bloqueo en redes sociales o listas de amigos.

Por su parte, Burke, Wallen, Vail-Smith y Knox (2011) pusieron el énfasis en el carácter sexual de las conductas de abuso online. Estos autores proponen una clasificación que distingue entre comportamientos de carácter sexual (p.ej., el envío de fotos íntimas y/o sexuales de la pareja sin permiso) y aquellos que no tienen ese carácter (p.ej., insultos, amenazas) a través de diferentes herramientas electrónicas.

\section{La evaluación del abuso online en la pareja}

La investigación sobre los instrumentos para evaluar el abuso online en la pareja ha avanzado considerablemente. El listado de los instrumentos elaborados para este fin hasta la fecha se presenta en la Tabla 2. Se han agrupado en función de si evalúan victimización (Bennett et al., 2011), perpetración (Lyndon, Bonds-Raacke y Cratty, 2011), o ambos (Borrajo, et al., 2015c). Como puede observarse, la mayoría de las escalas se centran en tipos específicos del abuso online en relaciones de noviazgo, como, por ejemplo, las conductas de control excesivo a través de Facebook (Tokunaga, 2011). Es importante señalar que algunos estudios no proporcionan evidencias sobre la validez de las escalas, o la evidencia sobre su validez es limitada. La mayoría de ellos sí informan sobre una adecuada fiabilidad, principalmente sobre la consistencia interna de las escalas.

Aunque es necesario avanzar en la evaluación del abuso online en la pareja, como puede observarse en la Tabla 2, contamos con una diversidad de instrumentos con adecuadas garantías de fiabilidad y validez.

\section{RECOMENDACIONES PARA LA PREVENCIÓN DEL ABUSO ONLINE EN LA PAREJA}

La evidencia empírica acumulada hasta el momento ha indicado las potenciales consecuencias que ser víctima del abuso 


\begin{tabular}{|c|c|c|}
\hline \multicolumn{3}{|c|}{$\begin{array}{c}\text { TABLA } 1 \\
\text { DENOMINACIONES PROPUESTAS AL FENÓMENO DEL ABUSO ONLINE EN LA PAREJA }\end{array}$} \\
\hline Autor (es) & Denominación & Definición o conductas \\
\hline Zweig et al. (2013) & Abuso cibernético en el noviazgo & $\begin{array}{l}\text { Ciber abuso sexual: presionar a la pareja para que envíe fotos sexuales o desnuda; envío de fotos } \\
\text { sexuales de la pareja a otros sabiendo que esta no quiere; amenazar a la pareja si no envía fotos } \\
\text { sexuales o desnuda; envío de mensajes de texto, mail o chats para mantener sexo o participar en } \\
\text { actos sexuales con la pareja sabiendo que ella no quiere. Ciber abuso no-sexual: Envío de mensajes } \\
\text { amenazantes; utilización de la red social de la pareja sin permiso; tomar un vídeo de la pareja y } \\
\text { enviarlo a amigos sin su permiso; envío de mensajes (SMS, chat, email) que le hagan sentir insegura; } \\
\text { utilizar los medios electrónicos para amenazar a la pareja físicamente; escribir en la red social de la } \\
\text { pareja cosas desagradables. }\end{array}$ \\
\hline $\begin{array}{l}\text { Schnurr, Mahatmya y } \\
\text { Basche (2013) }\end{array}$ & Ciber agresiones & "Conductas llevadas a cabo como represalias inmediatas a un daño percibido" \\
\hline $\begin{array}{l}\text { Association Press/MTV; } \\
\text { Liz Claiborne Inc. (2011) }\end{array}$ & Violencia digital en el noviazgo & $\begin{array}{l}\text { Comprobar dónde y con quién está la pareja múltiples veces al día; leer los mensajes sin permiso; } \\
\text { hacer borrar a la expareja de las listas de amigos de las redes sociales; insultarle a través de Internet } \\
\text { o el teléfono móvil; conocer las contraseñas sin permiso; contactar con la pareja para mantener } \\
\text { relaciones sexuales cuando ella no quiere; extender rumores sobre la pareja; utilizar la información } \\
\text { publicada en Internet contra la pareja para humillarla o avergonzarla; amenazar a la pareja con } \\
\text { hacerle daño. }\end{array}$ \\
\hline Melander (2010b) & Ciber acoso en pareja & $\begin{array}{l}\text { Adaptación de la tipología de Jonhson (2006) de violencia en pareja a un entorno online: } \\
\text { Violencia situacional en la pareja: Las nuevas tecnologías como precursores de episodios violentos } \\
\text { (p.ej., ojear el teléfono de la pareja). } \\
\text { Terrorismo íntimo: Conductas de control (p.ej., control constante de donde está la pareja y qué hace). } \\
\text { Control violento mutuo: Control mutuo a través de las nuevas tecnologías. } \\
\text { Resistencia violenta: Utilización de las nuevas tecnologías en defensa a las agresiones de la pareja } \\
\text { (p.ej., romper la relación a través de teléfono móvil). }\end{array}$ \\
\hline Draucker y Martsolf (2010) & $\begin{array}{l}\text { Agresiones electrónicas } \\
\text { Definición propuesta por David- } \\
\text { Ferdon y Hertz (2007) en el } \\
\text { contexto de las agresiones } \\
\text { electrónicas entre iguales. }\end{array}$ & $\begin{array}{l}\text { "Cualquier tipo de acoso o bullying, incluyendo provocaciones, mentiras, burlas, hacer comentarios } \\
\text { groseros o crueles, difundir rumores o hacer comentarios agresivos o amenazadores, que ocurren a } \\
\text { través de email, sala de chat, mensajería instantánea, páginas web o mensajes de texto". }\end{array}$ \\
\hline Bennet et al. (2011) & Victimización Electrónica & $\begin{array}{l}\text { Hostilidad electrónica: Publicación o envío de mensajes amenazantes, insultantes o dañinos a través } \\
\text { de redes sociales, mensajes de texto o mail. } \\
\text { Intrusividad: Control de correo electrónico y redes sociales, cambio de contraseñas y creación de un } \\
\text { perfil falso. } \\
\text { Humillaciones electrónicas: Publicación de fotos o información en redes sociales o webs para humillar } \\
\text { o avergonzar a la víctima. } \\
\text { Exclusión electrónica: Eliminación, exclusión o bloqueo en redes sociales o listas de amigos. }\end{array}$ \\
\hline Leisring y Giumetti (2014) & Ciber abuso psicológico & $\begin{array}{l}\text { Ciber abuso menor: insultar; dejar abruptamente de enviar mensajes o correos electrónicos durante } \\
\text { una discusión; utilización de letras mayúsculas para gritar; conseguir contraseñas revisando el correo } \\
\text { electrónico, mensajes del teléfono móvil o mensajes de las redes sociales. } \\
\text { Ciber abuso severo: amenazar; enviar correos electrónicos a otros sobre la pareja para humillarla o } \\
\text { avergonzarla; publicación de fotos inapropiadas de la pareja o información comprometida para } \\
\text { humillarla. }\end{array}$ \\
\hline $\begin{array}{l}\text { Cutbush, Williams, Miller, } \\
\text { Gibbs y Clinton-Sherrod } \\
\text { (2012) }\end{array}$ & Agresión electrónica en parejas & $\begin{array}{l}\text { Adaptado de Picard (2007). Insultar o decir cosas desagradables a la pareja; contactar con la pareja } \\
\text { cuando ella no quiere; hacer sentir miedo a la pareja; expandir rumores sobre la pareja; mostrar } \\
\text { fotos/vídeos privados o embarazosos a otros; amenazar con hacer daño a la pareja físicamente; } \\
\text { controlar repetidamente a la pareja para saber dónde está. }\end{array}$ \\
\hline
\end{tabular}


online podría provocar (Brown y Hegarty, 2018). Es por ello que urge desarrollar e implementar programas de prevención entre adolescentes y jóvenes adultos. Teniendo en cuenta los datos empíricos con los que contamos hasta este momento, cabe señalar tres recomendaciones fundamentales para el diseño de estrategias de prevención: 1) integrar la prevención de los dos tipos de violencia en la pareja, online y offline; 2) trabajar el papel de las actitudes que justifican la violencia; y 3) considerar la perpetración y la victimización como fenómenos relacionados.

\section{Integrar la prevención del abuso online con los programas de prevención de violencia en la pareja}

Estudios realizados entre adolescentes han encontrado que el abuso online en la pareja tiende a ocurrir junto con la violen- cia psicológica y física offline. Hinduja y Patchin (2011) encontraron que aquellos que se habían involucrado en formas de violencia tradicional admitían haberlo hecho también en sus formas electrónicas. Estos autores indicaron, además, que cabe identificar algunas conductas de riesgo que podrían derivar en la victimización, como compartir las contraseñas personales con la pareja.

Los resultados informados por Zweig et al. (2013) también siguen esta línea, indicando que la violencia online se relaciona con las diferentes formas de violencia tradicional (física, psicológica y sexual), tanto en cuanto a victimización como a perpetración. Así, la victimización de la violencia online se asoció con la victimización de las formas de violencia y abuso tradicionales, y la perpetración, por su parte, con la perpetración de las formas de violencia tradicional.

\begin{tabular}{|c|c|c|c|c|}
\hline \multicolumn{5}{|c|}{$\begin{array}{c}\text { TABLA } 2 \\
\text { EVIDENCIAS PSICOMÉTRICAS DE LOS INSTRUMENTOS UTILIZADOS EN ESTUDIOS }\end{array}$} \\
\hline \multicolumn{5}{|l|}{ Victimización } \\
\hline Autor (es) & $\begin{array}{l}\text { Denominación del } \\
\text { instrumento }\end{array}$ & Número de ítems y dimensiones & Validez & Fiabilidad \\
\hline Barter et al. (2017) & $\begin{array}{l}\text { Online Interpersonal } \\
\text { Violence and Abuse }\end{array}$ & $\begin{array}{l}6 \text { ítems que evalúan } 4 \text { formas principales de violencia y } \\
\text { abuso interpersonal a través de las nuevas tecnologías: } \\
\text { abuso emocional, conductas de control, vigilancia y } \\
\text { aislamiento. }\end{array}$ & & $\alpha: .76-.86$ \\
\hline Bennet et al. (2011) & Electronic victimization & 22 ítems: hostilidad intrusión, humillación, exclusión. & & $\begin{array}{l}\text { Hostilidad ( } \alpha=.74) \text {; Intrusión ( } \alpha=.73 \text { ); } \\
\text { Humillación }(\alpha=.74) \text {; Exclusión }(\alpha=.77)\end{array}$ \\
\hline $\begin{array}{l}\text { Carlson Fripp, Cook y } \\
\text { Kelchner (2015) }\end{array}$ & & $\begin{array}{l}5 \text { ítems adaptados de Schnurr, Mahatmy y Basche (2013) } \\
\text { desde Draucker and Martsolf (2010) }\end{array}$ & & $\alpha=.54$ \\
\hline Dick et al. (2014) & Cyber Dating Abuse & $\begin{array}{l}7 \text { ítems modificados de Ybarra, Espelage y Mitchell } \\
\text { (2007) y Bennett et al., (2011) Incluye dos categorías de } \\
\text { ítems:Ciber abuso sexual en pareja y ciber abuso no } \\
\text { sexual en pareja. }\end{array}$ & AFE & $\alpha=.72$ \\
\hline $\begin{array}{l}\text { Domínguez-Mora, } \\
\text { Vargas-Jiménez, Castro- } \\
\text { Castañeda y Nuñez- } \\
\text { Fadda (2016) }\end{array}$ & $\begin{array}{l}\text { Victimización en Redes } \\
\text { Sociales }\end{array}$ & 6 ítems que incluyen conductas de control. & & $\alpha=.90$. \\
\hline $\begin{array}{l}\text { Jaen-Cortés, Rivera- } \\
\text { Aragón, Reidl-Martínez } \\
\text { y García-Méndez } \\
\text { (2017). }\end{array}$ & $\begin{array}{l}\text { Escala de Violencia de } \\
\text { Pareja que se Expresa a } \\
\text { través de Medios } \\
\text { Electrónicos }\end{array}$ & $\begin{array}{l}32 \text { ítems } \\
5 \text { dimensiones: Control, vigilancia intrusiva y ciber } \\
\text { vigilancia (10) } \\
\text { Agresión verbal (11) } \\
\text { Agresión sexual (5) } \\
\text { Coerción sexual (3) } \\
\text { Humillación (3) }\end{array}$ & AFE & $\begin{array}{l}\alpha=.94 \text { para la escala total } \\
\alpha=.78-.93 \text { para las subescalas }\end{array}$ \\
\hline $\begin{array}{l}\text { Spitzberg y Hoobler } \\
\text { (2002) }\end{array}$ & $\begin{array}{l}\text { Cyber Obsessional } \\
\text { Pursuit Scale }\end{array}$ & $\begin{array}{l}24 \text { ítems sobre la frecuencia con la que la pareja lleva a } \\
\text { cabo conductas de persecución. } \\
3 \text { dimensiones } \\
\text { Hiper intimidad } \\
\text { Transferencia a la vida real } \\
\text { Amenazas }\end{array}$ & AFE & $\alpha=.77-.88$ \\
\hline
\end{tabular}




\begin{tabular}{|c|c|c|c|c|}
\hline \multicolumn{5}{|c|}{$\begin{array}{c}\text { TABLA } 2 \\
\text { EVIDENCIAS PSICOMÉTRICAS DE LOS INSTRUMENTOS UTILIZADOS EN ESTUDIOS SOBRE ABUSO ONLINE EN LA PAREJA (CONTINUACIÓN) }\end{array}$} \\
\hline \multicolumn{5}{|l|}{ Victimización } \\
\hline Autor (es) & $\begin{array}{l}\text { Denominación del } \\
\text { instrumento }\end{array}$ & Número de ítems y dimensiones & Validez & Fiabilidad \\
\hline $\begin{array}{l}\text { Wolford-Clevenger et } \\
\text { al. (2016) }\end{array}$ & $\begin{array}{l}\text { The Partner Cyber } \\
\text { Abuse Questionnaire }\end{array}$ & $\begin{array}{l}9 \text { ítems de Hamby (2013), incluyendo acoso, vigilancia, } \\
\text { humillación, y abuso verbal a través de las tecnologías, } \\
\text { como el teléfono móvil, redes sociales o correo } \\
\text { electrónico, perpetrado por la actual pareja. }\end{array}$ & AFE & $\alpha=.72$ \\
\hline \multicolumn{5}{|l|}{ Perpetración } \\
\hline Autor (es) & $\begin{array}{l}\text { Denominación del } \\
\text { instrumento }\end{array}$ & Número de ítems y dimensiones & Validez & Fiabilidad \\
\hline Fox y Warber (2013) & $\begin{array}{l}\text { Interpersonal Electronic } \\
\text { Surveillance for Social } \\
\text { Networking Sites, } \\
\text { adaptada de Tokunaga } \\
\text { (2011) }\end{array}$ & 13 ítems sobre vigilancia a través de redes sociales & AFE y AFC & $\alpha=.97$ \\
\hline Lyndon et al. (2011) & Facebook Survey & 13 ítems: provocación encubierta, acoso público, venting & AFE & $\alpha=.79-.88$ \\
\hline $\begin{array}{l}\text { Korchmaros, Ybarra, } \\
\text { Langhinrichsen-Rohling, } \\
\text { Boyd y Lenhart (2013) }\end{array}$ & $\begin{array}{l}\text { Perpetration in Dating } \\
\text { Relationships Scales, } \\
\text { adaptado de } \\
\text { Victimization in Dating } \\
\text { Relationships Scales } \\
\text { (Foshee et al., 1996) }\end{array}$ & 4 ítems: Control, celos, degradación & & \\
\hline $\begin{array}{l}\text { Sánchez, Muñoz- } \\
\text { Fernández y Ortega- } \\
\text { Ruíz (2015) }\end{array}$ & Cyberdating Q A & $\begin{array}{l}6 \text { dimensiones, } 2 \text { de ellas relacionadas con el abuso } \\
\text { online en pareja: control online ( } 6 \text { ítems) y conductas } \\
\text { intrusivas online ( } 4 \text { ítems) }\end{array}$ & AFE y AFC & $\alpha=.84-.85$ \\
\hline Schnurr et al. (2013) & $\begin{array}{l}\text { Cyber Aggression } \\
\text { Perpetration }\end{array}$ & 5 ítems adaptados de Draucker y Martsolf (2010). & & $\begin{array}{l}\alpha=.76 \text { y } .71 \text { para hombres } \text { y mujeres, } \\
\text { respectivamente }\end{array}$ \\
\hline $\begin{array}{l}\text { Tokunaga (2011) } \\
\text { [Modificada de Fox y } \\
\text { Warber (2013)] }\end{array}$ & $\begin{array}{l}\text { Interpersonal Electronic } \\
\text { Surveillance for Social } \\
\text { Networking Sites }\end{array}$ & $\begin{array}{l}12 \text { ítems que describen conductas de vigilancia y control } \\
\text { en redes sociales. }\end{array}$ & AFE y AFC & $\alpha=.97$ \\
\hline Wright (2015) & $\begin{array}{l}\text { Partner Direct Cyber- } \\
\text { Aggression }\end{array}$ & $\begin{array}{l}5 \text { ítems adaptados de un cuestionario que mide } \\
\text { agresiones relacionales en la pareja en el cara a cara } \\
\text { (Linder, Crick y Collins, 2002). } \\
2 \text { dimensiones: } \\
\text { Ciber agresiones relacionales (3) } \\
\text { Invasión de la privacidad (2) }\end{array}$ & AFC & $\alpha=.82-.91$ \\
\hline \multicolumn{5}{|l|}{$\begin{array}{l}\text { Perpetración y } \\
\text { victimización }\end{array}$} \\
\hline Autor (es) & $\begin{array}{l}\text { Denominación del } \\
\text { instrumento }\end{array}$ & Número de ítems y dimensiones & Validez & Fiabilidad \\
\hline Borrajo et al. (2015c) & $\begin{array}{l}\text { Cyber Dating Abuse } \\
\text { Questionnaire }\end{array}$ & $\begin{array}{l}20 \text { ítems paralelos } \\
\text { Agresión directa (1 1) } \\
\text { Control (9) }\end{array}$ & AFE y AFC & $\begin{array}{l}\alpha=.73-.84 \text { para perpetración, } \\
\alpha=.81-.87 \text { para victimización }\end{array}$ \\
\hline
\end{tabular}




\begin{tabular}{|c|c|c|c|c|}
\hline \multicolumn{5}{|c|}{$\begin{array}{c}\text { TABLA } 2 \\
\text { EVIDENCIAS PSICOMÉTRICAS DE LOS INSTRUMENTOS UTILIZADOS EN ESTUDIOS SOBRE ABUSO ONLINE EN LA PAREJA (CONTINUACIÓN) }\end{array}$} \\
\hline \multicolumn{5}{|l|}{$\begin{array}{l}\text { Perpetración y } \\
\text { victimización }\end{array}$} \\
\hline Autor (es) & $\begin{array}{l}\text { Denominación del } \\
\text { instrumento }\end{array}$ & Número de ítems y dimensiones & Validez & Fiabilidad \\
\hline Burke et al. 2011 & $\begin{array}{l}\text { Controlling Partner } \\
\text { Inventory (CPI) }\end{array}$ & $\begin{array}{l}18 \text { ítems: Fotos, cámara, GPS y/o Spyware, excesiva } \\
\text { comunicación, amenazas, conductas de control }\end{array}$ & AFE & $\alpha=.90$ \\
\hline $\begin{array}{l}\text { Celis-Sauce y Rojas- } \\
\text { Solis (2015) }\end{array}$ & $\begin{array}{l}\text { Ciber-violencia en el } \\
\text { Noviazgo [Cyberviolence } \\
\text { in Dating] }\end{array}$ & $\begin{array}{l}4 \text { ítems paralelos que miden agresiones psicológicas } \\
\text { como control, vigilancia y videovigilancia. }\end{array}$ & & $\begin{array}{l}\alpha=.74 \text { y } .43 \text { para perpetración y } \\
\text { victimización, respectivamente. }\end{array}$ \\
\hline Chaulk y Jones (2011) & $\begin{array}{l}\text { Online Obsessive } \\
\text { Relational Intrusion }\end{array}$ & 12 ítems: conductas benévolas, dañinas, peligrosas & & $\alpha=.71$ \\
\hline $\begin{array}{l}\text { Dank, Lachman, Zweig } \\
\text { y Yahner (2014) }\end{array}$ & Cyber Dating Abuse & $\begin{array}{l}16 \text { ítems adaptados de Picard (2007) y } 10 \text { de Griezel } \\
\text { (2007). } \\
\text { Items que miden abuso online en el noviazgo por la } \\
\text { actual pareja o la más reciente. }\end{array}$ & & \\
\hline $\begin{array}{l}\text { Duran y Martínez- } \\
\text { Pecino (2015) }\end{array}$ & $\begin{array}{l}\text { Escalas de Ciberacoso } \\
\text { contra la Pareja a través } \\
\text { del Teléfono Móvil y de } \\
\text { Internet }\end{array}$ & $\begin{array}{l}\text { Adaptada a las relaciones románticas de Victimization } \\
\text { Scales (Buelga, Cava y Musitu, 2010). } \\
\text { Dos dimensiones: } \\
\text { Ciber acoso utilizando teléfonos móviles, Ciber acoso } \\
\text { utilizando Internet }\end{array}$ & & $\begin{array}{l}\alpha=.75 \text { para la escala de perpetración } \alpha \\
\alpha=.62-.70 \text { para la escala de } \\
\text { victimización }\end{array}$ \\
\hline $\begin{array}{l}\text { Leisring y Giumetti } \\
\text { (2014) }\end{array}$ & $\begin{array}{l}\text { Cyber Psychological } \\
\text { Abuse (CPA) Scale }\end{array}$ & $\begin{array}{l}18 \text { ítems: Agresiones online leves y agresiones online } \\
\text { graves }\end{array}$ & AFE y AFC & $\begin{array}{l}\alpha=.81 \text { victimización; } \\
\alpha=.82 \text { perpetración } \\
\geq .70 \text { agresiones online leves y graves }\end{array}$ \\
\hline $\begin{array}{l}\text { Morelli, Bianchi, } \\
\text { Baiocco, Pezzuti y } \\
\text { Chirumbolo (2017) }\end{array}$ & $\begin{array}{l}\text { The Cyber Dating } \\
\text { Violence Inventory }\end{array}$ & $\begin{array}{l}22 \text { ítems paralelos adaptados de ítems del CADRI (Wolfe } \\
\text { et al., 2001). } \\
2 \text { dimensiones: } \\
\text { Ciber agresiones psicológicas en el noviazgo } \\
\text { Ciber agresiones relacionales en el noviazgo }\end{array}$ & AFE y AFC & $\begin{array}{l}\alpha=.81-.82 \text { para las escalas de } \\
\text { perpetración } \alpha=.82 \text { para las escalas } \\
\text { de victimización }\end{array}$ \\
\hline Muñiz (2017) & $\begin{array}{l}\text { Teen Dating Violence in } \\
\text { Social Networks Scale }\end{array}$ & $\begin{array}{l}10 \text { items that assess violent behaviors exhibited toward } \\
\text { the partner and former partner over the Internet. } \\
2 \text { dimensiones: } \\
\text { Actos violentos } \\
\text { Actos de control }\end{array}$ & AFE y AFC & $\alpha=.80-.86$ \\
\hline Reed et al. (2016) & $\begin{array}{l}\text { Digital Dating Abuse } \\
\text { Measure }\end{array}$ & $\begin{array}{l}19 \text { ítems paralelos que miden abuso, que implican } \\
\text { patrones de conducta como control, presiones, acoso, } \\
\text { amenazas u otros daños a la pareja, a través de } \\
\text { teléfonos móviles, ordenadores y comunicación por } \\
\text { Internet. }\end{array}$ & & $\begin{array}{l}\alpha=.76 \text { y } .73 \text { para victimización y } \\
\text { perpetración, respectivamente }\end{array}$ \\
\hline $\begin{array}{l}\text { Shorey, Cornelius, y } \\
\text { Strauss (2015) }\end{array}$ & $\begin{array}{l}\text { Stalking in Intimate } \\
\text { Relationships }\end{array}$ & $\begin{array}{l}6 \text { ítems sobre ciber acoso en una medida general de } \\
\text { acoso en relaciones de noviazgo }\end{array}$ & & $\begin{array}{l}\alpha=.65 \text { para perpetración y } \alpha=.63 \\
\text { para victimización }\end{array}$ \\
\hline Temple et al. (2016) & Cyber Abuse & $\begin{array}{l}13 \text { ítems paralelos modificados y adaptados de estudios } \\
\text { previos (Zweig et al. 2013; Picard 2007): }\end{array}$ & & $\begin{array}{l}\alpha=.65-.67 \text { para perpetración y } \\
\alpha=.74-.79 \text { para victimización }\end{array}$ \\
\hline Zweig et al. (2013) & Cyber dating abuse & $\begin{array}{l}16 \text { ítems paralelos: abuso online sexual, abuso online no } \\
\text { sexual }\end{array}$ & & $\begin{array}{l}\text { Abuso online sexual (victimización, } \\
\alpha=.81 \text {; perpetración, } \alpha=.88 \text { ) } \\
\text { Abuso online no sexual (victimización, } \\
\alpha=.89 \text {; perpetración, } \alpha=.92 \text { ) }\end{array}$ \\
\hline
\end{tabular}


Respecto a estudios realizados con muestras de jóvenes universitarios, Melander (2010a) encontró que la perpetración del abuso online en el noviazgo se asocia significativamente con una mayor perpetración de formas de violencia tradicional (física, psicológica y sexual). Igualmente, la victimización de las agresiones electrónicas también mostró relación positiva con la violencia tradicional.

En resumen, los resultados de las diversas investigaciones muestran que ambos tipos de agresiones (online y offline) tienden a relacionarse y a compartir factores de riesgo comunes. En base a la evidencia empírica señalada, la prevención e intervención en uno y otro tipo de abuso debe integrarse de una manera holística.

\section{Trabajar las actitudes que justifican el abuso online}

El papel de las creencias que justifican la violencia como factor de riesgo ha sido ampliamente evaluado tanto en la violencia general (Hinduja y Patchin, 2011; Zweig et al., 2013) como en la violencia en parejas (Calvete, 2008; FernándezGonzález, Calvete y Orue, 2017b; Huesmann y Guerra, 1997). Además, los programas desarrollados para prevenir e intervenir sobre la violencia offline en la pareja han hecho especial énfasis sobre las creencias como posible factor de riesgo en la aparición de estas conductas (Muñoz-Rivas, Gámez-Guadix, Fernández-González y González-Lozano, 2011).

Las creencias justificadoras de la violencia hacia la pareja están muy extendidas entre los jóvenes. Por ejemplo, Muñoz-Rivas, Graña, O'Leary, y González (2007) hallaron, en una muestra de jóvenes entre 16 y 20 años, que aproximadamente el $13 \%$ los hombres justificaban las agresiones cuando eran realizadas en defensa propia, mientras que el $22 \%$ de las mujeres en momentos emocionales de una intensa furia o enfado. Respecto al abuso online en el noviazgo, Borrajo, GámezGuadix y Calvete (2015b) encontraron que las actitudes que justifican las agresiones incrementaron la probabilidad de agresión directa a través de las TICs (p.ej., amenazar o insultar a la pareja).

Por otro lado, son diversos los autores que han señalado las creencias distorsionadas sobre el amor como un factor de riesgo para la aparición de la violencia en relaciones de noviazgo y las han incorporado en los esfuerzos para prevenir este problema (Garrido Genovés y Tello, 2009). Algunos autores han indicado que los jóvenes podrían ser especialmente vulnerables a una mala interpretación de la violencia en la pareja debido a la visión irreal y distorsionada que tienen del amor (Sharpe y Taylor, 1999). Se ha sugerido incluso que la idea de que "el amor todo lo puede" podría provocar la disminución de disonancia cognitiva y crear la esperanza de que las agresiones desaparecerán (González-Ortega, Echeburúa y Corral, 2008). Estas ideas irracionales o mitos incluyen la creencia sobre la existencia de una persona perfecta para cada uno, la creencia de que los celos son una muestra de amor o la creencia en que querer a alguien da derecho a abusar de esa persona (p.ej., controlando todo lo que hace) (Ferrer Pérez y Bosch Fiol, 2013).
En España, son diversos los estudios que han hallado la amplia aceptación que estas creencias sobre el amor tienen entre los jóvenes. Por ejemplo, Marroquí y Cervera (2014) encontraron que alrededor del $30 \%$ de una muestra de jóvenes estaban de acuerdo o totalmente de acuerdo con el mito de la media naranja (existe una pareja perfecta para cada uno). Asimismo, más del $70 \%$ indicó estar de acuerdo con la creencia de que el amor lo puede todo. Ferrer, Bosch, y Navarro (2010) encontraron también una alta prevalencia de aceptación de los mitos sobre el amor en una muestra de rango de edad más amplio (18-93 años). En el rango referido a jóvenes de entre 18 y 34 años, hallaron que alrededor del $80 \%$ se mostraba de acuerdo con alguna creencia distorsionada sobre el amor (p.ej., los celos son una muestra de amor). Los resultados mostraron, además, que eran las mujeres quienes presentaban mayores prevalencias en relación a los mitos sobre el poder del amor (p.ej., el amor debe poderlo todo). Sin embargo, los hombres mostraron mayor acuerdo con los mitos sobre la importancia de la pareja y estar emparejado (p.ej., "la separación de la pareja es un fracaso").

Por tanto, en base a esta evidencia empírica y a la considerable extensión de las actitudes que justifican las agresiones en relaciones de pareja, este debe ser un punto central de los programas de prevención.

\section{La perpetración y la victimización constituyen fenómenos relacionados}

La investigación sobre violencia offline física y psicológica en relaciones de adolescentes y jóvenes ha encontrado sistemáticamente que la perpetración y la victimización tienden a aparecer relacionadas (p.ej., Fernández-González, Calvete y Orue, 2017a). En otras palabras, la víctima suele ser también agresor, y el agresor tiende a convertirse en víctima. Estos resultados han sido también informados para el abuso online (Leisring y Giumetti, 2014; Reed et al., 2017).

Estos hallazgos conllevan una serie de implicaciones para la prevención. En primer lugar, la prevención debería ir enfocada a intervenir en los factores que favorecen la reciprocidad entre perpetración y victimización. En este sentido, es necesario que los programas preventivos proporcionen estrategias para aprender a manejar situaciones específicas que podrían facilitar la aparición de estos comportamientos. Los celos o el enfado parecen presentarse como importantes precursores en la aparición del abuso online, por lo que es importante detectar estas situaciones y favorecer estrategias para interpretarlas y gestionarlas de manera adecuada sin recurrir a comportamientos agresivos (Borrajo et al., 2015a).

En segundo lugar, se debe evitar el etiquetaje de los adolescentes que participan en los programas de prevención (p.ej., "el maltratador"). Los datos sobre la prevalencia de conductas como el control, las amenazas y/o las humillaciones a través de herramientas electrónicas parecen mostrar que estas conductas forman parte de la comunicación habitual de las parejas jóvenes (Kellerman, Margolin, Borofsky, Baucom y Iturralde, 2013), alcanzado tasas del $80 \%$. Por tanto, el empleo de tér- 
minos estigmatizantes podría resultar contraproducente. Más bien, desde una perspectiva educativa, recomendamos identificar conductas inadecuadas que deben ser corregidas.

Finalmente, es necesario fomentar los factores protectores que incluyen la autoestima, la empatía, la asertividad y las estrategias de resolución de conflictos adecuadas, que redundarán, a su vez, en la prevención de la perpetración y la victimización.

\section{CONCLUSIONES}

El abuso online en la pareja ha comenzado recientemente a recibir atención como una forma diferenciada de violencia en la pareja. Se trata de una forma relativamente reciente de agresión y victimización, que requiere más investigación y esfuerzos adicionales para su prevención.

Los resultados sobre la prevalencia hallados en los estudios realizados muestran la alta incidencia que las agresiones online tienen en las relaciones de pareja, tanto en estudios nacionales (Borrajo, et al., 2015c) como internacionales (Lyndon et al., 2011). Esto nos urge a desarrollar estudios que nos permitan profundizar en las características y correlatos que se relacionan con este fenómeno, con el objetivo de adquirir un mayor conocimiento sobre el mismo y poder diseñar estrategias de prevención adecuadas. Sin embargo, aunque la atención sobre el abuso online en la pareja está aumentando de manera exponencial, las diferentes denominaciones propuestas para conceptualizar el fenómeno y la diversidad de instrumentos desarrollados para medirlo (Brown y Hegarty, 2018), ponen de manifiesto la necesidad de seguir avanzando en este campo.

Además, es escaso el conocimiento que tenemos sobre los factores de riesgo que propician su aparición, así como de las consecuencias de ser víctima. Por otro lado, de cara a obtener una perspectiva más amplia del fenómeno, es fundamental hacer hincapié en factores familiares (p.ej., experiencias de maltrato en la familia o exposición a la violencia en el hogar) y personales (baja autoestima, impulsividad, etc.) que pueden estar relacionados con la aparición de estas conductas. Esto permitirá conocer si los factores de riesgo asociados a la victimización y la perpetración del abuso online en parejas son similares a los hallados en la aparición de la violencia en parejas offline y los aparecidos en otras formas de acoso a través de las nuevas tecnologías, como el cyberbullying (GámezGuadix y Gini, 2016).

Una limitación importante a este respecto es que las investigaciones disponibles hasta el momento son de naturaleza transversal. Futuros estudios longitudinales deben examinar la relación temporal entre factores de riesgo, perpetración y victimización en el abuso online y consecuencias para el ajuste psicosocial.

Finalmente, es importante fomentar, desde el contexto educativo, familiar y social, la promoción del uso responsable de las TICs como herramientas que favorecen el desarrollo personal y la comunicación con otras personas, y la implementación sistemática de programas de prevención de la violencia en el contexto de la pareja, incluido el abuso que se produce a través de Internet y los Smartphones.

\section{REFERENCIAS}

Almendros, C., Gamez-Guadix, M., Antonio Carrobles, J., Rodriguez-Carballeira, A. y Porrua, C. (2009). Intimate partner psychological abuse: concept, measurement, and recent contributions. Psicologia Conductual, 17(3), 433-451.

Archer, J. (2000). Sex differences in aggression between heterosexual partners: a meta-analytic review. Psychological Bulletin, 126(5), 651.

Associated Press/MTV (2011). Associated Press-MTV digital abuse survey August 2011. Recuperado de: http://www.athinline.org/pdfs/201 1 MTVAP_Digital_Abuse_Study_Full.pdf.

Barter, C., Stanley, N., Wood, M., Lanau, A., Aghtaie, N., Larkins, C. y Øverlien, C. (2017). Young people's online and face-to-face experiences of interpersonal violence and abuse and their subjective impact across five European countries. Psychology of Violence, 7, 375-384. doi:10.1037/vio0000096

Bennett, D. C., Guran, E. L., Ramos, M. C. y Margolin, G. (2011). College students' electronic victimization in friendships and dating relationships: Anticipated distress and associations with risky behaviors. Violence and Victims, 26, 410.

Borrajo, E. y Gámez-Guadix, M. (2016). Cyber dating abuse: its link to depression, anxiety and dyadic adjustment. Psicologia Conductual, 24, 221-235.

Borrajo, E., Gámez-Guadix, M. y Calvete, E. (2015a). Cyber dating abuse: prevalence, context, and relationship with offline dating aggression. Psychological Reports, 116, 565585. doi:10.2466/21.16.PRO.116k22w4

Borrajo, E., Gámez-Guadix, M. y Calvete, E. (2015b). Justification beliefs of violence, myths about love and cyber dating abuse. Psicothema, 27(4), 327-333. doi:10.7334/psicothema2015.59

Borrajo, E., Gámez-Guadix, M., Pereda, N. y Calvete, E. (2015c). The development and validation of the cyber dating abuse questionnaire among young couples. Computers in Human Behavior, 48, 358-365. doi:10.1016/i.chb.2015.01.063

Bowen, E., Walker, K., Mawer, M., Holdsworth, E., Sorbring, E., Helsing, B., . . A Awouters, V. (2014). "It's like you're actually playing as yourself": Development and preliminary evaluation of 'Green Acres High', a serious game-based primary intervention to combat adolescent dating violence. Psychosocial Intervention, 23(1), 43-55.

Brown, C. y Hegarty, K. (2018). Digital dating abuse measures: A critical review. Aggression and Violent Behavior, 40, 44-59. doi: 10.1016/i.avb.2018.03.003

Burke, S. C., Wallen, M., Vail-Smith, K. y Knox, D. (2011). Using technology to control intimate partners: An exploratory study of college undergraduates. Computers in Human Behavior, 27(3), 1162-1167. doi:10.1016/i.chb.2010.12.010

Carlson, R. G., Fripp, J., Cook, C. y Kelchner, V. (2015). Examining intimate partner violence, stress and technology use among young adults. The Professional Counselor, 5(3), 365378. doi:10.15241/rgc.5.3.365 
Calvete, E. (2008). Justification of violence and grandiosity schemas as predictors of antisocial behavior in adolescents. Journal of Abnormal Child Psychology, 36, 1083-1095. doi:10.1007/s10802-008-9229-5

Celis-Sauce, A. y Rojas-Solís, J. (2015). Violencia en el noviazgo desde la perspectiva de varones adolescentes. Informes Psicológicos, 15, 83-104.

Chaulk, K. y Jones, T. (2011). Online obsessive relational intrusion: Further concerns about Facebook. Journal of Family Violence, 26, 245-254. doi:http://dx.doi.org/10.1007/s10896$011-9360-x$

Cutbush, S., Williams, J., Miller, S., Gibbs, D. y Clinton-Sherrod, M. (2012). Electronic dating aggression among middle school students: Demographic correlates and associations with other types of violence. Poster presented at the 140th American Public Health Association, annual meeting, October 27-31; San Francisco, CA (Recuperado de: http://www.rti.org/pubs/apha12_cutbush_poster.pdf)

Dank, M., Lachman, P., Zweig, J. M. y Yahner, J. (2014). Dating violence experiences of lesbian, gay, bisexual, and transgender youth. Journal of Youth and Adolescence, 43, 846-857. doi:10.1007/s10964-013-9975-8

Darvell, M. J., Walsh, S. P. y White, K. M. (2011). Facebook tells me so: Applying the theory of planned behavior to understand partner-monitoring behavior on Facebook. $\mathrm{Cy}^{-}$ berpsychology, Behavior, and Social Networking, 14, 717-722.

Dick, R. N., McCauley, H. L., Jones, K. A., Tancredi, D. J., Goldstein, S., Blackburn, S., ... y Miller, E. (2014). Cyber dating abuse among teens using school-based health centers. Pediatrics, 134, e1560-e1567.

Domínguez-Mora, R., Vargas-Jiménez, E., Castro-Castañeda, R. y Nuñez-Fadda, S. M. (2016). Impacto de la comunicación familiar en la victimización por internet en parejas adolescentes. Opción, 13, 979-1000.

Draucker, C. B. y Martsolf, D. S. (2010). The role of electronic communication technology in adolescent dating violence. Journal of Child and Adolescent Psychiatric Nursing, 23, 133-142. doi: 10.1111/j.1744-6171.2010.00235.x

Durán, M. y Martínez-Pecino, R. (2015). Ciberacoso mediante teléfono móvil e Internet en las relaciones de noviazgo entre jóvenes. Comunicar, 22, 159-167. doi:10.3916/C442015-17

Fernández-González, L., Calvete, E. y Orue, I. (2017a). Adolescent dating violence stability and mutuality: a 4 -year longitudinal study. Journal of Interpersonal Violence. doi: $10.1177 / 0886260517699953$

Fernández-González, L., Calvete, E. y Orve, I. (2017b). La escala Acceptance of Dating Violence (ADV): propiedades psicométricas de la versión española. Psicothema, 29(2), 241-246.

Ferrer Pérez, V. y Bosch Fiol, E. (2013). Del amor romántico a la violencia de género. Para una coeducación emocional en la agenda educativa. Profesorado, 17, 105-122.

Ferrer, V. A., Bosch, E. y Navarro, C. (2010). Los mitos román- ticos en España. Boletín de Psicología, 99, 7-31.

Fox, J., Osborn, J. L. y Warber, K. M. (2014). Relational dialectics and social networking sites: The role of Facebook in romantic relationship escalation, maintenance, conflict, and dissolution. Computers in Human Behavior, 35, 527-534. https://doi.org/10.1016/i.chb.2014.02.031

Fox, C.L. y Warber, K. (2014). Social networking sites in romantic relationships: Attachment, uncertainty, and partner surveillance on Facebook. Cyberpsychology, Behavior, and Social Networking, 7(1), 3-7. https://doi.org/10.1089/cyber.2012.0667

GÁmez-Guadix, M. y Gini, G. (2016). Individual and class justification of cyberbullying and cyberbullying perpetration: A longitudinal analysis among adolescents. Journal of $A p$ plied Developmental Psychology, 44, 81-89. doi:10.1016/j.appdev.2016.04.001

Garrido Genovés, V. y Tello, M. C. (2009). La prevención de la violencia en la relación amorosa entre adolescentes a través del taller "La Máscara del Amor». Revista de Educación, 349, 335-360.

González-Ortega, I., Echeburúa, E. y Corral, P. d. (2008). Variables significativas en las relaciones violentas en parejas jóvenes: una revisión. Psicología Conductual, 16(2), 207225.

Hinduja, S. y Patchin, J. (2011). Electronic dating violence: A brief guide for educators and parents. Cyberbullying Research Center. Recuperado de: https://cyberbullying.org/electronic_dating_violence_fact_sheet.pdf

Huesmann, L. R. y Guerra, N. G. (1997). Children's normative beliefs about aggression and aggressive behavior. Journal of Personality and Social Psychology, 72(2), 408.

Izaguirre, A. y Calvete, E. (2015). Children who are exposed to intimate partner violence: Interviewing mothers to understand its impact on children. Child Abuse \& Neglect, 48, 5867. doi: 10.1016/j.chiabu.2015.05.002

Jaen-Cortés, C. I., Rivera-Aragón, S., Reidl-Martínez, L. M. y García-Méndez, M. (2017). Violencia de pareja a través de medios electrónicos en adolescentes mexicanos. Acta de Investigación Psicológica, 7, 2593-2605. https://doi.org/10.1016/i.aipprr.2017.01.001

Kellerman, I., Margolin, G., Borofsky, L. A., Baucom, B. R. y Iturralde, E. (2013). Electronic aggression among emerging adults: Motivations and contextual factors. Emerging Adulthood, 1(4), 293-304. https://doi.org/10.1177/2167696813490159

Korchmaros, J. D., Ybarra, M. L., Langhinrichsen-Rohling, J., Boyd, D. y Lenhart, A. (2013). Perpetration of teen dating violence in a networked society. Cyberpsychology, Behavior, and Social Networking, 16, 561-567. doi: 10.1089/cyber.2012.0627

Leisring, P. A. y Giumetti, G. W. (2014). Sticks and stones may break my bones, but abusive text messages also hurt: Development and validation of the Cyber Psychological Abuse scale. Partner Abuse, 5, 323-341. doi:10.1891/19466560.5.3.323 
Lyndon, A., Bonds-Raacke, J. y Cratty, A. D. (2011). College students' Facebook stalking of ex-partners. Cyberpsychology, Behavior, and Social Networking, 14(12), 711-716. doi:10.1089/cyber.2010.0588

Marroquí, M. y Cervera, P. (2014). Interiorización de los falsos mitos del amor romántico en jóvenes. Reidocrea: Revista Electrónica de Investigación y Docencia Creativa, 3, 142146.

Melander, L. A. (2010a). College students' perceptions of intimate partner cyber harassment. Cyberpsychology, Behavior, and Social Networking, 13, 263-268. doi:10.1089=cyber.2009.0221

Melander, L.A. (2010b). Explaining college partner violence in the digital age: An instrumental design mixed methods study (Tesis Doctoral) Department of Psychology, University of $\mathrm{Ne}^{-}$ braska at Lincoln, Nebraska, United States. Recuperado de: http://digitalcommons.unl.edu/cgi/viewcontent.cgi?article$=1000$ \& context=sociologydiss.

Morelli, M., Bianchi, D., Baiocco, R., Pezzuti, L. y Chirumbolo, A. (2016). Not-allowed sharing of sexts and dating violence from the perpetrator's perspective: The moderation role of sexism. Computers in Human Behavior, 56, 163-169. doi: 10.1016/i.chb.2015.11.047

Muñiz, M. (2017). Online teen dating violence, family and school climate from a gender perspective/Violencia de pareja online en la adolescencia, clima familiar y escolar desde la perspectiva de género. Infancia y Aprendizaje, 1-27. doi:10.1080/02103702.2017.1341101

Muñoz-Rivas, M. J., Graña, J. L., O'Leary, K. D. y González, M. P. (2007). Aggression in adolescent dating relationships: Prevalence, justification, and health consequences. Journal of Adolescent Health, 40(4), 298-304. doi:10.1016/i.jadohealth.2006.11.137

Muñoz-Rivas, M., Gamez-Guadix, M., Fernandez-Gonzalez, L. y Gonzalez Lozano, M. P. (2011). Validation of the Attitudes About Aggression in Dating Situations (AADS) and the Justification of Verbal/Coercive Tactics Scale (JVCT) in Spanish Adolescents. Journal of Family Violence, 26(8), 575584. doi:10.1007/s10896-011-9391-3

Reed, L. A., Tolman, R. M. y Ward, L. M. (2017). Gender matters: Experiences and consequences of digital dating abuse victimization in adolescent dating relationships. Journal of Adolescence, 59, 79-89. doi:10.1016/i.adolescence.2017.05.015

Sánchez, V., Muñoz-Fernández, N. y Ortega-Ruíz, R. (2015). "Cyberdating Q_A": An instrument to assess the quality of adolescent dating relationships in social networks. Computers in Human Behavior, 48, 78-86. https://doi.org/10.1016/i.chb.2015.01.006

Schnurr, M. P., Mahatmya, D. y Basche, R. A., III. (2013). The role of dominance, cyber aggression perpetration, and gen- der on emerging adults' perpetration of intimate partner violence. Psychology of Violence, 3, 70-83. doi:10.1037/a0030601

Sharpe, D. y Taylor, J. K. (1999). An examination of variables from a social-developmental model to explain physical and psychological dating violence. Canadian Journal of Behavioural Science, 31(3), 165.

Spitzberg, B. H. y Hoobler, G. (2002). Cyberstalking and the technologies of interpersonal terrorism. New Media \& Society, 4, 71-92. doi: 10.1177/14614440222226271.

Stonard, K. E., Bowen, E., Lawrence, T. R. y Price, S. A. (2014). The relevance of technology to the nature, prevalence and impact of adolescent dating violence and abuse: $A$ research synthesis. Aggression and Violent Behavior, 19(4), 390-417. https://doi.org/10.1016/j.avb.2014.06.005

Tokunaga, R. S. (2011). Social networking site or social surveillance site? Understanding the use of interpersonal electronic surveillance in romantic relationships. Computers in Human Behavior, 27, 705-713. https://doi.org/10.1016/j.chb.2010.08.014

Vagi, K. J., Olsen, E. O. M., Basile, K. C. y Vivolo-Kantor, A. M. (2015). Teen dating violence (physical and sexual) among US high school students: Findings from the 2013 National Youth Risk Behavior Survey. JAMA pediatrics, 169(5), 474-482. doi:10.1001/jamapediatrics.2014.3577.

Vu, N. L., Jouriles, E. N., McDonald, R. y Rosenfield, D. (2016). Children's exposure to intimate partner violence: a meta-analysis of longitudinal associations with child adjustment problems. Clinical Psychology Review, 46, 25-33. https://doi.org/10.1016/i.cpr.2016.04.003

Wincentak, K., Connolly, J. y Card, N. (2017). Teen dating violence: A meta-analytic review of prevalence rates. Psychology of Violence, 7, 224-241. http://dx.doi.org/10.1037/a0040194

Wolford-Clevenger, C., Zapor, H., Brasfield, H., Febres, J., Elmquist, J., Brem, M., . . . Stuart, G. L. (2016). An examination of the partner cyber abuse questionnaire in a college student sample. Psychology of Violence, 6, 156-162. doi:http://dx.doi.org/10.1037/a0039442

Wright, M. F. (2015). Cyber aggression within adolescents' romantic relationships: Linkages to parental and partner attachment. Journal of Youth and Adolescence, 44(1), 37-47. doi:http://dx.doi.org/10.1007/s10964-014-0147-2

Zweig, J. M., Dank, M., Yahner, J. y Lachman, P. (2013). The rate of cyber dating abuse among teens and how it relates to other forms of teen dating violence. Journal of Youth and Adolescence, 42(7), 1063-1077.

Zweig, J. M., Lachman, P., Yahner, J. y Dank, M. (2014). Correlates of cyber dating abuse among teens. Journal of Youth and Adolescence, 43(8), 1306-1321. 